and at $100 \mathrm{kHz}$ electrical modulation, which demonstrated that the output signal tracked the input signal at transistor speed.

With a three-terminal HBT as a light source, a wide range of applications is possible for integrated circuits that would use both electrical and optical signals, including the interconnection of optical and electrical signals for display technologies and communication purposes. In addition, it could make this transistor the fundamental element in optoelectronics as well as in electronics. This work might be the start of an era in which photons are transported around a chip in the same fashion as electrons, according to the researchers.

Alfred A. ZINN

\section{Tailored Contrast Agent for Nuclear Spin Tomography Optimized}

Nuclear spin tomography (or magnetic resonance imaging, MRI) has developed into an important medical diagnostic procedure. Although MRI is often used in a non-invasive manner, the contrast between tissues of different types is sometimes too low for effective diagnosis. Consequently, materials are often used to increase the sensitivity or specificity of an image. A research team from the University of Texas at Dallas, Macrocyclics Inc., and the University of Texas Southwestern Medical Center has demonstrated optimization of contrast agents based upon complexes of the rare-earth metal gadolinium, as reported in the December 2003 issue of Angewandte Chemie International Edition.
Nuclear spin tomography uses the "spin" of hydrogen atom nuclei. The spins align themselves in a strong magnetic field, but a pulsed radio wave causes them to "flip" in the opposite direction. After the pulse, the hydrogen atoms release electromagnetic waves as they fall back to their ground spin state. The amplitude and duration of this signal depend on the concentration of hydrogen and the relaxation times, which differ for different tissue types, but may not differ enough for unambiguous analysis. Contrast agents, which influence the relaxation times of the protons in water molecules, are used to increase the contrast. Gadolinium ions have seven unpaired electrons, which induce a strong alternating electromagnetic field that "shakes" the spins of neighboring water molecules, causing them to return to their ground state more quickly than usual. However, gadolinium ions have toxic effects, and they need to be well protected within a complex. A. Dean Sherry of the University of Texas at Dallas and his team are using 1,4,7,10-tetra-azacyclododecane1,4,7,10-tetra-acetic acid complex with a

\section{FOR MORE RESEARCH NEWS ON MATERIALS SCIENCE ... \\ ... access the Materials Research Society Web site: \\ www.mrs.org/gateway/ \\ matl_news.html}

carbon-nitrogen ring with arms to contain the ion. The gadolinium ion is held fast by four nitrogen atoms from the ring and four oxygen atoms from the arms, and sits within these ligands. On the "open" side, there is room for one water molecule. In order for the contrast agent to function optimally, the water molecule must disappear as quickly as possible after "relaxing" so as to make room for another. The turnover time depends on the structure of the complex.

Each set of four nitrogen and four oxygen atoms in the complex makes a square. This results in two possible configurations, an antiprism and a twisted antiprism, which differ in the degree of rotation of the two squares relative to each other. These can be interconverted by a "flip" of the ring and a rotation of the arms. To determine whether the two forms hold onto water molecules for different lengths of time, Mark Woods of the University of Texas at Dallas attached "stoppers" to the ring and each arm in order to prevent flipping and rotation. By selecting the stereochemical configuration of the side-chain arms, both forms could be obtained. The researchers found that water molecules spent considerably less time in the twisted antiprism configuration than in the antiprism configuration. The rate of water exchange in the twisted antiprism was $15 \mathrm{~ns}$, close to the theoretical optimum value of $30 \mathrm{~ns}$. This represents an important step toward a targeted design for nuclear spin contrast agents, according to the research team. $\square$
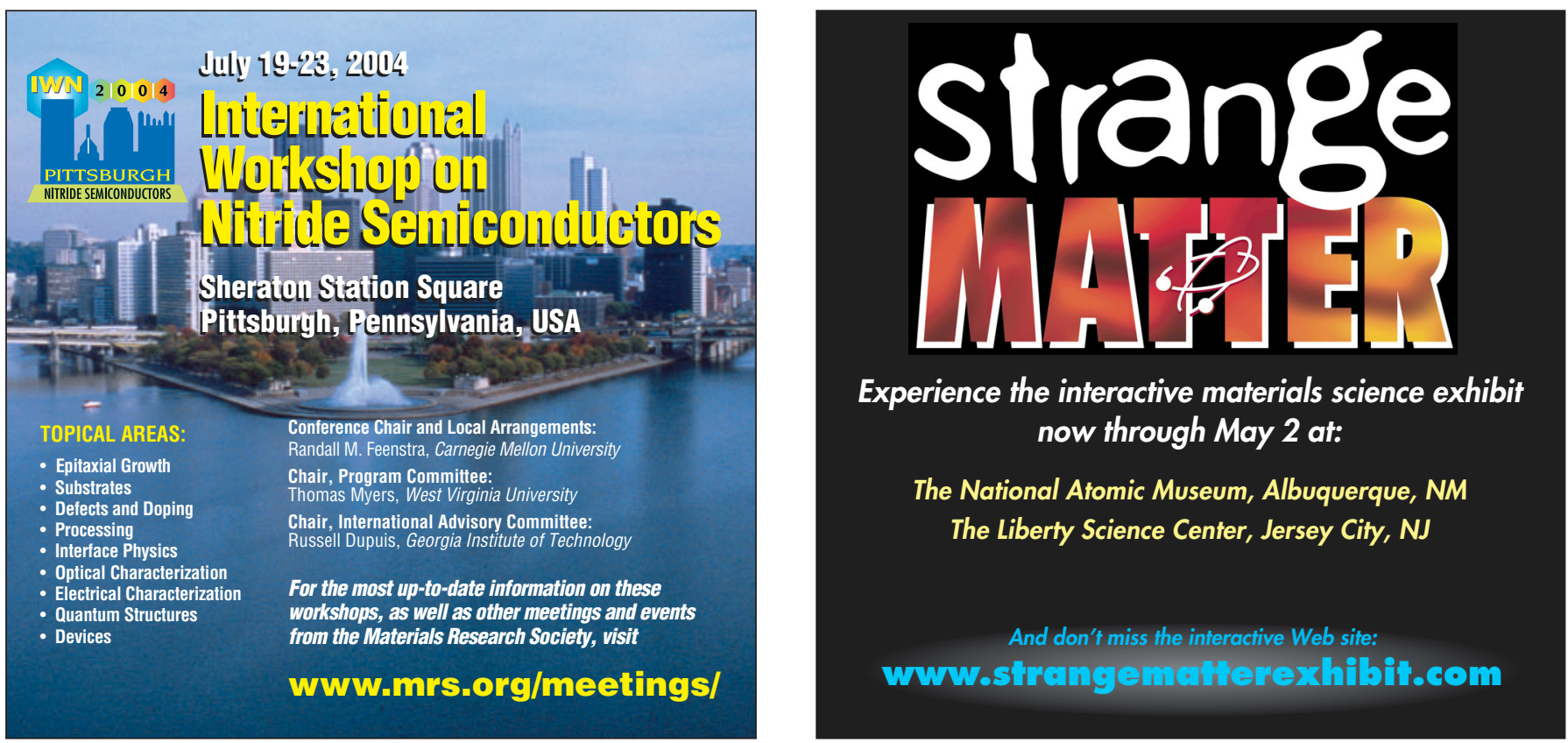